\title{
The Swiss Master in Nuclear Engineering: A Collaboration between Universities, Research Centre and Industry
}

R. Chawla a, b, J.P. Ansermet $^{\text {a }}$, J.M. Cavedon ${ }^{\text {b }}$, P. Hirt ${ }^{c}$, W. Kröger ${ }^{\text {, }}$, H.M. Prasser ${ }^{\text {d, b }}$, M.Q. Tran ${ }^{a}$

a EPFL, Lausanne, Switzerland

b PSI, Villigen, Switzerland

${ }^{\mathrm{c}}$ swissnuclear, Olten, Switzerland

d ETHZ, Zurich, Switzerland

ICONE18, Xi'an, China

May, 2010 


\section{Contents}

$\square$ Introduction

$\square$ Program Goals, Qualification Profile

$\square$ Curriculum Structure and Contents

$\square$ Organisational Aspects

$\square$ Experience to Date, Future prospects

$\square$ Conclusions 


\section{Introduction}

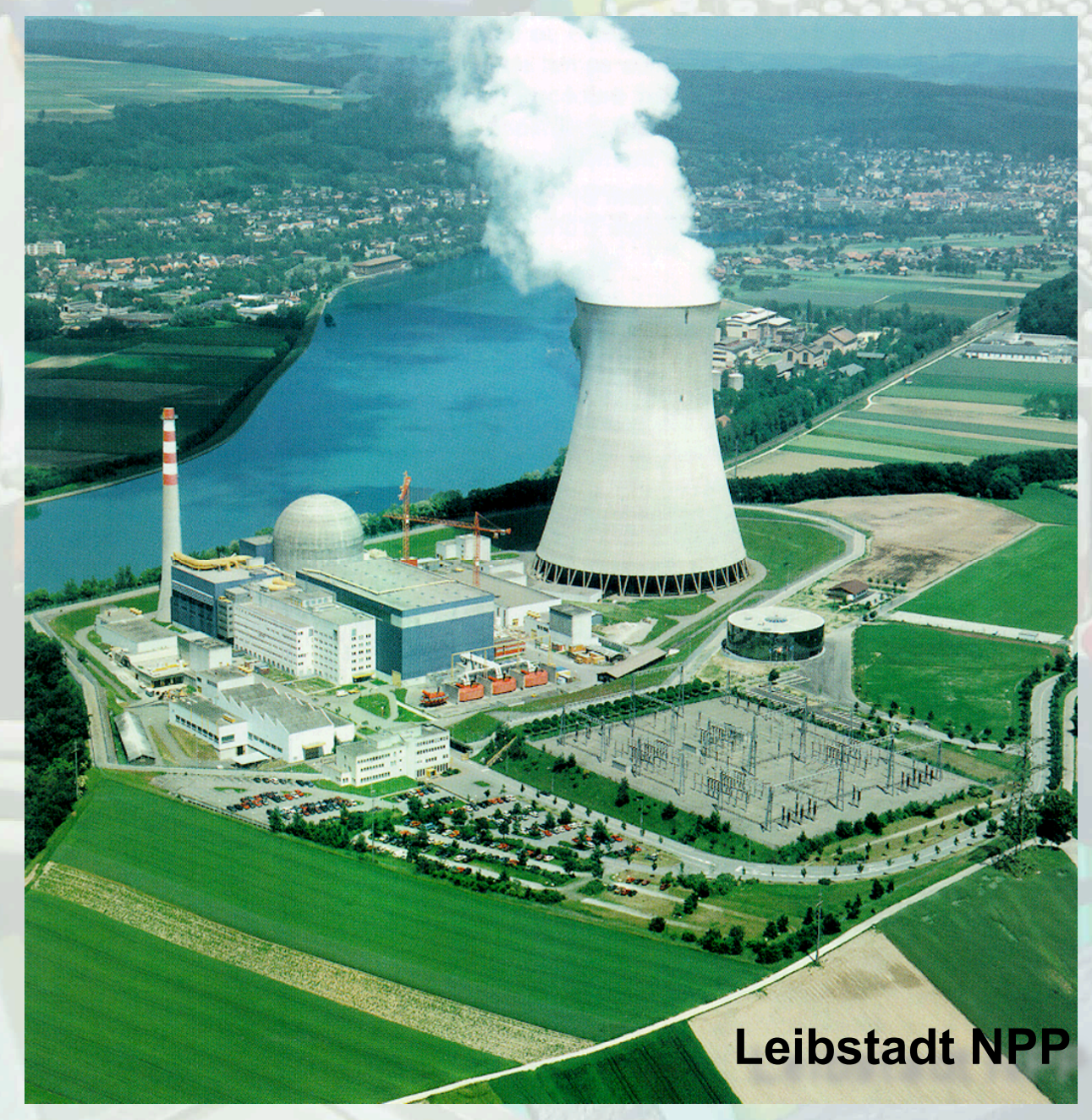

$\square 40 \%$ of electricity in Switzerland, nuclear

- Important option for future

- University-level education in field, crucial national need

S Swiss Federal Institutes of Technology: Lausanne (EPFL), Zurich (ETHZ)

- Long tradition of nuclear-related education

- Optional courses (physics, mech. eng.)

- $\mathrm{PhD}$ research, often with PSI collaboration

- Support of industry (swissnuclear)

$\square$ Pooling of resources recently for set-up of new Master of Science in Nucl. Eng. (NE)

- Present paper: main features, experience with running of this first-ever joint degree 


\section{Program Goals}

o Provide in-depth knowledge on nuclear energy fundamentals, technology

- Represents primary goal

- Strongly inter-disciplinary field implies wide range of subjects to be taught

- Provide complementary knowledge on nuclear fusion

- Strong background of fusion research at EPFL

o Provide knowledge on nuclear techniques in medicine, research and industry

- Optional courses in radioisotope applications, accelerator physics

o Provide view of entire fuel cycle, from uranium mining to the back-end

- Sustainability largely defined by fuel cycle, corresponding compulsory course

- Underline role of nuclear energy as part of a sustainable, global energy mix

- Several electives related to non-nuclear energy production 


\section{Qualification, Employment Prospects}

o Qualification profile corresponding to wide range of potential employers

- From utilities, via nuclear service companies, to NPP vendors and developers

o Thus, in Switzerland

- Nuclear utilities: Alpiq, Axpo, BKW-FMB

- Nuclear waste disposal organisations: Nagra, ZWILAG

- Other types of industrial employers mainly abroad

- Reactor vendors, fuel manufacturers, enrichment plants, reprocessing facilities

- R\&D organisations, both inland (PSI) and abroad (GRS, IRSN, ...)

- National nuclear safety authorities and other related government institutions

- Swiss Federal Nuclear Safety Inspectorate (ENSI), ...

- Non-nuclear fields, e.g. general energy sector, R\&D (fluids, materials,...) 


\section{Curriculum Structure}

o Student's achievements measured in ECTS credit points

- Full academic year: 60 ECTS, i.e. 1 ECTS $\sim 1 \mathrm{~h}$ class $+1 \mathrm{~h}$ homework per week

o Swiss NE Master currently 90 ECTS, i.e. 3-semester, program

- 2 semesters, course work; $3^{\text {rd: }}$ 8-week industrial internship + 17-week project

o EPFL-ETHZ-PSI-swissnuclear collaboration reflected by:

- $1^{\text {st }}$ semester fully at EPFL, $2^{\text {nd }}$ at ETHZ, $3^{\text {rd }}$ at PSI

o Overall structure:

- 28 ECTS compulsory courses, 20 core electives

- 4 ECTS free elective, 2 entrepreneurship course, 6 Semester project

- 30 ECTS Master thesis

o Teaching language: English 


\section{Compulsory Courses}

o Seven compulsories

- Each to be cleared separately

- First four at Lausanne

- EPFL: Physics, Materials

o Other three at Zurich

- ETHZ: Th'hydls, Engineering

o Several courses, "standard"

- Reactor Experiments, uses CROCUS reactor at EPFL

- Special Topics in Reactor Physics, advanced aspects

- Nuclear Systems, fuel cycle and impact on NPP operations

\begin{tabular}{|c|c|c|c|c|c|}
\hline No. & Course & \begin{tabular}{|c|} 
Responsible \\
University
\end{tabular} & Semester & Held at & ECTS \\
\hline 1 & Neutronics & EPFL & Autumn & Lausanne & 4 \\
\hline 2 & $\begin{array}{l}\text { Reactor } \\
\text { Experiments }\end{array}$ & EPFL & Autumn & Lausanne & 4 \\
\hline 3 & Reactor Technology & ETHZ & Autumn & Lausanne & 4 \\
\hline 4 & $\begin{array}{l}\text { Nuclear Fuels and } \\
\text { Materials }\end{array}$ & EPFL & Autumn & Lausanne & 4 \\
\hline 5 & Nuclear Safety & ETHZ & Spring & Zurich & 4 \\
\hline 6 & $\begin{array}{l}\text { Special Topics in } \\
\text { Reactor Physics }\end{array}$ & EPFL & Spring & Zurich & 4 \\
\hline 7 & $\begin{array}{l}\text { Nuclear Energy } \\
\text { Systems }\end{array}$ & ETHZ & Spring & Zurich & 4 \\
\hline & & & & Total: & 28 \\
\hline
\end{tabular}




\section{Core Electives - 1}

O 20 ECTS of core courses (5 electives), chosen from three different tracks"

- A. Energy Systems

- B. Physics \& Materials

- C. Thermal-hydraulics

o Tracks, not specializations, solely to guide students in their choice

o Advice also provided by "tutor"
Track A: Energy Systems

\begin{tabular}{|c|l|c|c|c|}
\hline No. & Course & $\begin{array}{c}\text { Responsible } \\
\text { University }\end{array}$ & Semester & Held at \\
\hline 1 & $\begin{array}{l}\text { Advanced Fossil and } \\
\text { Renewable Energy Systems }\end{array}$ & EPFL & Autumn & Lausanne \\
\hline 2 & Hydraulic Turbomachines & EPFL & Autumn & Lausanne \\
\hline 3 & $\begin{array}{l}\text { Probabilistic Safety } \\
\text { Analysis and Risk } \\
\text { Management for Critical } \\
\text { Energy Infrastructure }\end{array}$ & ETHZ & Spring & Zurich \\
\hline 4 & $\begin{array}{l}\text { Renewable Energy } \\
\text { Technologies II, Energy } \\
\text { Storage and Conversion }\end{array}$ & ETHZ & Spring & Zurich \\
\hline
\end{tabular}

ICONE18, Xi'an, May 2010.. 8 


\section{Core Electives - 2}

Track B: Physics and Materials

Track C: Thermal-hydraulics

\begin{tabular}{|c|l|c|c|c|}
\hline No. & Course & $\begin{array}{c}\text { Responsible } \\
\text { University }\end{array}$ & Semester & Held at \\
\hline 1 & $\begin{array}{l}\text { Nuclear Fusion and Plasma } \\
\text { Physics }\end{array}$ & EPFL & Autumn & Lausanne \\
\hline 2 & $\begin{array}{l}\text { Introduction to Particle } \\
\text { Accelerators }\end{array}$ & EPFL & Autumn & Lausanne \\
\hline 3 & $\begin{array}{l}\text { Radioisotope and Radiation } \\
\text { Applications }\end{array}$ & EPFL & Spring & Zurich \\
\hline 4 & $\begin{array}{l}\text { Advanced Topics in Nuclear } \\
\text { Reactor Materials }\end{array}$ & EPFL & Spring & Zurich \\
\hline
\end{tabular}

\begin{tabular}{|c|l|c|c|c|}
\hline 1 & Instability and Turbulence & EPFL & Autumn & Lausanne \\
\hline 2 & $\begin{array}{l}\text { Two-Phase Flows and Heat } \\
\text { Transfer }\end{array}$ & EPFL & Autumn & Lausanne \\
\hline 3 & $\begin{array}{l}\text { Multi-Phase Thermal Fluid } \\
\text { Dynamics }\end{array}$ & ETHZ & Spring & Zurich \\
\hline 4 & Advanced CFD Methods & ETHZ & Spring & Zurich \\
\hline
\end{tabular}




\section{Semester Project}

o Introduction to nuclear R\&D

o 1 day per week during $2^{\text {nd }}$ sem.

- Preparation for Master thesis

o Basis for choice of topic:

- Course work during $1^{\text {st }}$ sem.

- Advice of tutor

- Visit to PSI at end of $1^{\text {st }}$ sem. (national centre for nuclear R\&D), site for Master research

- Presentation of main R\&D activities underway in NES Dept., e.g. in Dec.'09

\begin{tabular}{|c|c|c|}
\hline No. & $R \& D$ Activity & $\begin{array}{l}\text { Responsible } \\
\text { Laboratory }\end{array}$ \\
\hline 1. & Experimental Reactor Physics & \multirow{3}{*}{$\begin{array}{l}\text { Reactor Physics and } \\
\text { Systems Behaviour }\end{array}$} \\
\hline 2. & LWR Core \& Transient Analysis & \\
\hline 3. & Gen. IV Fast Reactor Studies & \\
\hline 4. & Thermal-hydraulic Phenomena & \multirow[t]{2}{*}{ Thermal-hydraulics } \\
\hline 5. & Severe Accident Phenomena & \\
\hline 6. & Nuclear Fuels R\&D & \multirow[t]{3}{*}{ Nuclear Materials } \\
\hline 7. & Reactor Component Safety & \\
\hline 8. & High Temperature Materials & \\
\hline 9. & Analytical Techniques (Fuels) & Hot Lab \\
\hline 10. & Waste Management & Waste Management \\
\hline 11. & Technology Assessment & \multirow[t]{2}{*}{ Energy Systems } \\
\hline 12. & Risk \& Human Reliability & \\
\hline 13. & Materials for Nuclear Fusion & \multirow{2}{*}{$\begin{array}{l}\text { Centre for Research in } \\
\text { Plasma Physics (EPFL) }\end{array}$} \\
\hline 14. & Magnets for Nuclear Fusion & \\
\hline
\end{tabular}




\section{Master Project}

o Master project (30 ECTS): two parts

- 8-week industrial internship (NPPs, ENSI, Nagra, etc.)

- 17-week Master thesis, usually built upon semester project

o Master thesis

- Enhance capability for independent theoretical and/or experimental NE research

- Condition for starting: clearance of at least 50 ECTS of course work (from total of 60 needed)

- Supervision: senior PSI/NES scientist + responsible professor

Examples of chosen research topics, 2008 batch $\rightarrow$

\begin{tabular}{|c|l|}
\hline 1. & HELIOS analysis of a SCWR-like test lattice in PROTEUS \\
\hline 2. & Improved Monte Carlo calculations of RPV fluence \\
\hline 3. & Analysis of PHENIX pre-shutdown tests \\
\hline 4. & Feasibility study for neutron tomography of 2-phase flows \\
\hline 5. & Validation of CFD modelling results \\
\hline 6. & Modelling of reactor containment flows \\
\hline 7. & An isotopic dilution technique for fission gas analysis \\
\hline 8. & LCA analysis of waste disposal and $\mathrm{CO}_{2}$ sequestration \\
\hline
\end{tabular}




\section{Organisational Aspects: Core Group, Tutors}

o Program conducted under supervision of NE "Core Group" (NECG)

- Professors from each university, PSI-NES department head, swissnuclear representative, ...

- Decisions on academic matters by professors (formal ratification by both universities)

o Each student chooses a tutor at start of program

- Academic mentor, advisor (e.g. on choice of optional courses)

o Many of the courses taught by professors, but not all

- Additional lecturers needed for meeting demands of wide-based curriculum

- Necessary academic personnel, largely from among senior PSI-NES scientists

- Approval from university department through NECG professor 


\section{Admission Requirements, Procedure}

o Program open to wide range of Bachelor degree holders

- Mechanical/Electrical/Chemical Engineering, Materials Science, Physics, Chemistry, Maths.

- Justified by multi-disciplinary character of NE Master

- Common admission profile, corresponding to $\sim$ first 2 years of Bachelor

- 18 ECTS Mathematics, 12 ECTS Natural Sciences, 12 ECS Engineering Sciences

o Evaluation of candidates on basis of detailed dossiers

- NE Core Group, role of Admission Committee (AC)

- Principal criteria: degree, parent university, academic performance

o Several decisions taken on individual basis

- Subsidiary course requirements, if necessary

- Allocation of students from abroad to the 2 different universities (registration) 


\section{Administration, Mobility Support, Degree}

o Admission / registration for courses, 2 independent procedures

- All students registered at EPFL for $1^{\text {st }}$ semester, at ETHZ for $2^{\text {nd }}$

- Exchange student status for those admitted at "other" university

- Creditation of courses, responsibility of host university

o Semester, Master projects

- Responsibility with EPFL or ETHZ professor, depending on topic (may or may not be tutor)

o Mobility support granted by ETH-Domain's energy competence centre (CCEM-CH)

- Funding to cover extra expenditure related to "multi-campus" nature of program

o Degree issued jointly by the 2 universities

- Master of Science EPF-ETH in Nuclear Engineering 


\section{Experience to Date}

o First 2 batches of similar size

- 12 students in 2008 (4 from Swiss universities, 8 from abroad)

- 13 students in 2009 (7 from Swiss universities, 6 from abroad)

- Large fraction of students from abroad, reflection of "Bologna spirit"

o Bachelor degrees of various types (among the 25 students to date)

- Physics: 11, Mech. Eng.: 6, Nucl. Eng.: 2, Chem. Eng.: 2, Others: 4

- Domination of Physics, Mech. Eng., in line with "host" departments at EPFL, ETHZ

o Feedback from students, generally very positive

- Attending classes in 2 different universities with different "cultures", enriching experience

- Despite "mixed" background of students, courses found both interesting and challenging

- Wide range of R\&D topics offered at PSI, felt to be a noteworthy strength 


\section{Future Prospects}

o Current 90 ECTS (3 semester) program would benefit from upgrade to 120 ECTS

- Make course work less "dense"

- Allow greater flexibility in structuring industrial internship, Master project

- Render program fully compatible with " 3 + 2"-year Bologna system

o Deliberations during 2009, decision to implement new curriculum starting this Sept.

- Main change: additional, new $3^{\text {rd }}$ semester at PSI for semester project and 4 "block" courses

NE core (block) courses in $3^{\text {rd }}$ semester of new 120 ECTS curriculum

\begin{tabular}{|c|l|c|c|c|c|}
\hline No. & Course & $\begin{array}{c}\text { Responsible } \\
\text { University }\end{array}$ & Course Type & $\begin{array}{c}\text { Held } \\
\text { at }\end{array}$ & ECTS \\
\hline 1 & $\begin{array}{l}\text { Radiobiology and } \\
\text { Radiation Protection }\end{array}$ & ETHZ & $\begin{array}{c}\text { Compulsory } \\
\text { (new) }\end{array}$ & PSI & 4 \\
\hline 2 & $\begin{array}{l}\text { Adv. Topics in Nucl. } \\
\text { Reactor Materials }\end{array}$ & EPFL & Core Elective & PSI & 4 \\
\hline 3 & System Codes Lab & EPFL & $\begin{array}{c}\text { Core Elective } \\
\text { (new) }\end{array}$ & PSI & 3 \\
\hline 4 & $\begin{array}{l}\text { Beyond-Design- } \\
\text { Basis Safety }\end{array}$ & ETHZ & $\begin{array}{c}\text { Core Elective } \\
\text { (new) }\end{array}$ & PSI & 3 \\
\hline \multicolumn{7}{|l}{} & Total: & 14 \\
\hline
\end{tabular}




\section{Conclusions}

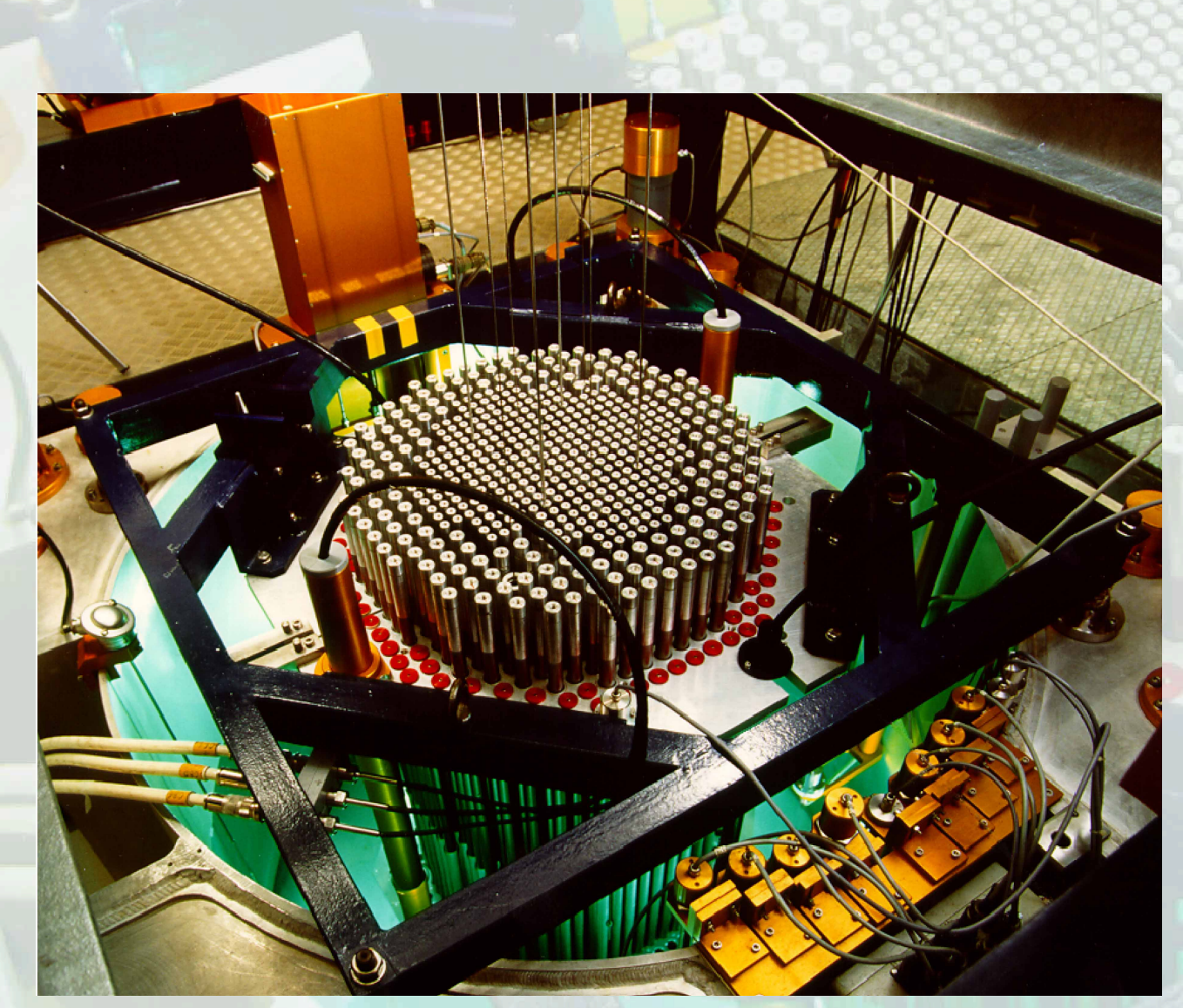

Reactor CROCUS at EPFL
- As first-ever common EPFL- ETHZ degree, Swiss NE Master a unique collaboration between universities, research centre and industry

- Although mainly addressing national needs, to be viewed in international context

- Reflected in high fraction (> 50\%) of students from abroad

- Program upgrade to 4 semesters from Sept. 2010 onwards

o Further strengthening of curriculum

- Greater conformity with other NE programs, increased prospects for international collaborations 


\section{Thank you for your attention!}

